\title{
Effects of Geometric Factors and Material Properties on Stress Behavior in Rotating Disk
}

\author{
Vahid Monfared ${ }^{1 *}$, Mohammadhassan Hassan ${ }^{2}$, Saeed Daneshmand ${ }^{3}$, Farshad Taheran ${ }^{4}$ and Reza \\ Ghaffarivardavagh $^{5}$ \\ 'Department of Mechanical Engineering, Zanjan Branch, Islamic Azad University, \\ Zanjan, Iran; vahid.monfared@iauz.ac.ir \\ ${ }^{2}$ Department of Mechanical Engineering, Sharif University of Technology, \\ PO Box 11365-9567,Tehran, Iran; mh.hasan.1990@gmail.com \\ ${ }^{3}$ Department of Mechanical Engineering, Majlesi Branch, Islamic Azad University, \\ Isfahan, Iran; s.daneshmand@iaumajlesi.ac.ir \\ ${ }^{4}$ School of Mechanical Engineering, Sharif University of Technology, \\ Tehran, Iran; F_Taheran@mech.sharif.edu \\ ${ }^{5}$ School of Mechanical Engineering, Sharif University of Technology, Tehran, Iran; rezaghaffari@alum.sharif.edu
}

\begin{abstract}
In this study, effects of geometric factors and material properties are investigated on stress behavior of circular rotating disk with constant rotation in various industrial mechanisms using equilibrium equations, geometric relations and stress functions. In this way, research on a thin uniform and homogeneous circular disk under constant rotation is considered. The rotating motion produces centrifugal acceleration on each element of the rotating disk, and this rotating motion becomes the source of external loading for the mentioned problem. Additional exterior loadings are not assumed in this problem. It is comfortable to handle the centrifugal force loading by relating it to a body force density via the disk in order to increase strength (maximum stress) of circular rotating disk. Eventually, stress behavior of rotating disk is analyzed with considering geometric factors and material properties analytically. Finally, it is concluded that the mentioned factors affect on increasing and decreasing stress values of circular rotating disk under constant rotation.
\end{abstract}

Keywords: Centrifugal Acceleration, Equilibrium Equations, Rotating Disk.

\section{Introduction}

Determination of stress behavior in rotating disk is an essential problem which has received much attention. The mentioned problem was first worked out mainly in connection with steam turbines. Recent development of industrial machines for moving gases (blowers, fans, superchargers for airplane engines) has led to an increased interest in the subject and has introduced special requirements. Also, this problem is of interest of many engineers in industrial world. Ehrenfest Paradox relates the rotating inflexible disc in the theory of relativity. In its original and innovative formulation as proposed ${ }^{1}$, it discusses a perfectly rigid cylinder that is made to rotate about its axis of symmetry. Many numerical and analytical methods and approaches have been performed for obtaining strength of the rotating disk and its stress behavior under different conditions by various investigators $^{2-10}$. For example, analytical and numerical approaches are also employed for stress-strain analysis of the rotating disks with non-uniform thickness and density like, Variable Material Properties (VMP) Theory and Numerical Runge-Kutta's Method for elasto-plastic regions with considering elastic-linear hardening [4]. Furthermore, Jafari et al. ${ }^{8}$ designed optimum disk profiles for minimum weight

*Author for correspondence: 
by the Karush-Kuhn-Tucker Method (KKT) as a classical optimization method, Simulated Annealing (SA) and Particle Swarm Optimization (PSO) as two contemporary optimization methods. Moreover, experimental attempts have been also carried out for analyzing rotating disk by many researchers ${ }^{11-14}$. For instance, an experimental work was also undertaken on the turbulent flow because of an enclosed rotational disk, in which, measurements of the average velocity distributions and all six elements of Reynolds Stress were made for the two boundary layers developed on the rotational disk and the steady state end wall ${ }^{11}$. It should be mentioned that other kinds of researches were performed in the similar fields such as PZT materials (PZT-lead $(=\mathrm{Pb})$ zirconate $(=\mathrm{Zr}$ ) titanate $(=\mathrm{Ti})$ or $\mathrm{Pb}[\mathrm{ZrxTi1}-\mathrm{x}] \mathrm{O}_{3}$. It is mixture of $\mathrm{PbTiO}_{3}$ and $\mathrm{ZrTiO}_{3}$, used in rotating disk and rotating wheel analysis in railway engineering ${ }^{15,16}$. Accordingly, it can say that rotating disk analysis have high importance in engineering sciences. Therefore, it should be analyzed carefully for obtaining correct behavior of rotating disk in constant rotation.

Analysis of the rotating disk, rotating MHD (MagnetoHydro-Dynamic) viscous flow, rotating system studies, three-dimensional rotating flow using Spline method and other similar analyses about rotating systems have been performed in fluids and viscous materials ${ }^{17-20}$. Moreover, flow of a linearly viscous fluid between two rotating disks about two different vertical axes has been investigated. In which, an estimated theoretical solution has been determined by taking into account the case of rotation with a small angular velocity difference ${ }^{17}$. It should be noted that some references have been used for such parameters as elastic modulus, atomic number and so on ${ }^{21,29}$. In addition, some studies have been done about engineering and mathematical problems for more investigation of such engineering and mathematical problems which can help to researchers ${ }^{22-28}$.

In this work, stress behaviors of the rotating disk are predicted using equilibrium equations, geometric relations and stress functions analytically. So, stress analysis is carried out in order to determine critical regions in the rotating disk. In which, stress in the rotating disk is studied with considering the material and geometrical changes.

\section{Materials and Method}

In this section, a slender uniform and homogeneous circular disk is assumed subjected to a constant rotation $\omega$ as shown in Figure 1. One of the applications of this research is simply in disk sanders analysis. Figure 1 simulates

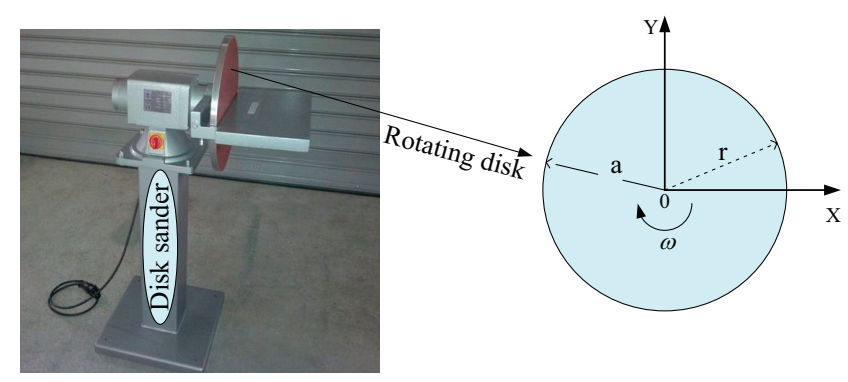

Figure 1. Rotating circular disk under angular velocity $\omega$.

rotating system situated in the disk sander with simple disk rotating as below.

The rotational motion creates centrifugal acceleration on each element of the rotating disk, and this subsequently becomes the resource of exterior loading for the mentioned problem. Also, other extra exterior loadings aren't considered. It is useful and serviceable to manage the centrifugal force loading by relating it to a body force density and other related parameters through the disk in order for good designing of rotating machines. For the case of constant angular velocity, the body force in radial direction is given by

$$
\begin{aligned}
& \sum_{i=1}^{n} F_{i}=F_{1}+F_{2}+F_{3}+\ldots+F_{n}=m \frac{d v}{d t}=m \dot{v}=m a \\
& F_{r}=m a_{r}=\rho r \omega^{2}
\end{aligned}
$$

where, $\rho$ is the material mass density. Next, equilibrium equations in the cylindrical coordinate are introduced in general form as below,

$$
\begin{aligned}
& \frac{\partial \sigma_{r}}{\partial r}+\frac{1}{r} \frac{\partial \tau_{r \theta}}{\partial \theta}+\frac{\partial \tau_{r z}}{\partial z}+\frac{1}{r}\left(\sigma_{r}-\sigma_{\theta}\right)+F_{r}=0 \\
& \frac{\partial \tau_{r \theta}}{\partial r}+\frac{1}{r} \frac{\partial \sigma_{\theta}}{\partial \theta}+\frac{\partial \tau_{\theta z}}{\partial z}+\frac{2}{r} \tau_{r \theta}+F_{\theta}=0 \\
& \frac{\partial \tau_{r z}}{\partial r}+\frac{1}{r} \frac{\partial \tau_{\theta z}}{\partial \theta}+\frac{\partial \sigma_{z}}{\partial z}+\frac{1}{r} \tau_{r z}+F_{z}=0
\end{aligned}
$$

This problem is related to the axisymmetric model, and subsequently the equilibrium equations reduce to the following form,

$$
\frac{\partial \sigma_{r}}{\partial r}+\frac{1}{r}\left(\sigma_{r}-\sigma_{\theta}\right)+F_{r}=0
$$

The solution may be powerfully handled utilizing a special stress function that automatically satisfies the equilibrium equations. The particular stress function relation with this property is given by the following form,

$$
\sigma_{\theta}=\frac{d \Phi}{d r}+r F_{r}
$$




$$
\sigma_{r}=\frac{\Phi}{r}
$$

where, $\Phi=\Phi(r)$ is stress function. Also, strain fields in the cylindrical coordinate are given as below general forms,

$$
\begin{aligned}
\varepsilon_{r} & =\frac{\partial u_{r}}{\partial r} \\
\varepsilon_{\theta} & =\frac{1}{r}\left(u_{r}+\frac{\partial u_{\theta}}{\partial \theta}\right) \\
\varepsilon_{z} & =\frac{\partial u_{z}}{\partial z} \\
\varepsilon_{r \theta} & =\frac{1}{2}\left(\frac{1}{r} \frac{\partial u_{r}}{\partial \theta}+\frac{\partial u_{\theta}}{\partial r}-\frac{u_{\theta}}{r}\right) \\
\varepsilon_{\theta z} & =\frac{1}{2}\left(\frac{\partial u_{\theta}}{\partial z}+\frac{1}{r} \frac{\partial u_{z}}{\partial \theta}\right) \\
\varepsilon_{z r} & =\frac{1}{2}\left(\frac{\partial u_{r}}{\partial z}+\frac{\partial u_{z}}{\partial r}\right)
\end{aligned}
$$

In this problem, $u_{r}=u_{r}(r)$ and $u_{\theta}=0$. Therefore it can be given as,

$$
\begin{aligned}
\varepsilon_{r} & =\frac{\partial u_{r}}{\partial r} \\
\varepsilon_{\theta} & =\frac{1}{r}\left(u_{r}\right) \\
\varepsilon_{r \theta} & =0
\end{aligned}
$$

By combining equations $9-17$, compatibility relation is determined,

$$
\begin{gathered}
\frac{\partial}{\partial r}\left(r \varepsilon_{\theta}\right)-\varepsilon_{r}=0 \\
\varepsilon_{\theta}+r \frac{\partial \varepsilon_{\theta}}{\partial r}-\varepsilon_{r}=0
\end{gathered}
$$

Using Hook’s Law gives,

$$
\begin{aligned}
\varepsilon_{r} & =\frac{1}{E}\left(\sigma_{r}-v \sigma_{\theta}\right)=\frac{1}{E}\left(\frac{\Phi}{r}-v \frac{d \Phi}{d r}-v \rho \omega^{2} r^{2}\right) \\
\varepsilon_{\theta} & =\frac{1}{E}\left(\sigma_{\theta}-v \sigma_{r}\right)=\frac{1}{E}\left(\frac{d \Phi}{d r}+\rho \omega^{2} r^{2}-v \frac{\Phi}{r}\right)
\end{aligned}
$$

In which, $\mathrm{E}$ and $v$ are respectively Elastic Modulus and Poisson's Ratio. Substituting equations 19 and 20 into equation 18 , gives,

$$
\frac{d^{2} \Phi}{d r^{2}}+\frac{1}{r} \frac{d \Phi}{d r}-\frac{\Phi}{r^{2}}+(3+v) \rho r \omega^{2}=0
$$

By integrating equation 21, we get,

$$
\begin{aligned}
& \int\left(\int \frac{d}{d r}\left(\frac{1}{r} \frac{d(r \Phi)}{d r}\right) d r\right) d r= \\
& -\int\left(\int(3+v) \rho r \omega^{2} d r\right) d r+c_{1} r+c_{2} \\
& \Phi=-\frac{(v+3)}{8} \rho r^{2} \omega^{2}+\frac{c_{1}}{2}+\frac{c_{2}}{r^{2}}
\end{aligned}
$$

Therefore stresses are obtained by above formulations, that is,

$$
\begin{aligned}
& \sigma_{r}=\frac{\Phi}{r}=-\frac{(v+3)}{8} \rho r^{2} \omega^{2}+\frac{c_{1}}{2}+\frac{c_{2}}{r^{2}} \\
& \sigma_{\theta}=\frac{d \Phi}{d r}+r F_{r}=-\frac{(3 v+1)}{8} \rho r^{2} \omega^{2}+\frac{c_{1}}{2}-\frac{c_{2}}{r^{2}}
\end{aligned}
$$

In above equations, if $r=0$, then $\sigma=\infty$, that is not acceptable due to bounded stresses. Also, stresses are zero at external edges $r=a$. So, $c_{1}=0.25(v+3) \rho a^{2} \omega^{2}$ and $c_{2}=0$. Therefore stress behavior of rotating circular disk is given as,

$$
\begin{aligned}
& \sigma_{r}=\frac{3+v}{8} \rho \omega^{2}\left(a^{2}-r^{2}\right) \\
& \sigma_{\theta}=\frac{\rho \omega^{2}}{8}\left[(v+3) a^{2}-(3 v+1) r^{2}\right]
\end{aligned}
$$

It should be mentioned that the maximum stress values occur at the center of the disk where $F_{\mathrm{r}}$ is equal to zero. The maximum stress is given by the following,

$$
\sigma_{\max }=\left.\sigma_{r}\right|_{r=0}=\left.\sigma_{\theta}\right|_{r=0}=\frac{v+3}{8} \rho a^{2} \omega^{2}
$$

\section{Results and Discussions}

Aluminum (Al) and Steel are considered for comparison of the stress values and their behaviors in the rotating disk with considering geometric factors. Properties of material behaviors are available in references ${ }^{21,29}$. Now, stress behaviors in the rotating disk are analyzed for aluminum (Al) and TS40000 series stainless steel with considering geometric factor effects.

As mentioned before, material properties of assumed materials have been presented in references ${ }^{21,29}$. In what follows, results of this work have been presented in the following figures (Figures 2-6). First, stress behaviors in the rotating circular disk are presented for Aluminum Circular Disk with considering geometric factors and material properties shown in Figures 2 and 3. The following 
results can be benefit and profit in many industrial machines and mechanisms.

In Figure 2, abrupt and sudden changes are seen in the stress behaviors in the rotating disk for angular velocity $20 \mathrm{rad} / \mathrm{s}$ with respect to radius changes at the center of the disk in aluminum unlike the other cases. This behavior decrease general stability of the rotating disk and is unfavorable.

This behavior isn't suitable for the rotating disk and should be considered in mechanical design of aluminum rotating disk.

In Figure 3, smooth and mellow changes and slopes are found in the aluminum stress behavior in the rotating disk in angular velocity between $15 \mathrm{rad} / \mathrm{s}$ to $20 \mathrm{rad} / \mathrm{s}$ at the center of the disk on the contrary the other intervals. This behavior is caused by high stability of the rotating disk in the mentioned regions. This trend is also proper for the rotating disk and should be noted in the mechanical design of aluminum rotating disk with various radius. It then follows that, behavior of the rotating circular disk is presented with considering geometric factors and material characteristics in steel (TS40000 series stainless steel) shown in Figures 4 and 5.

In Figures 4 and 5, gradient changes of steel stress behavior in the rotating disk are more sudden and abrupt than the aluminum stress behavior in the rotating disk at the center of the disk. It must be considered in the mechanical design of the rotating disk. Therefore, results of maximum stress in the rotating disk under constant angular velocity in Aluminum rotating disk have been compared with steel (TS40000 series stainless steel) rotating circular disk with considering various geometric factors and angular velocity shown in Figure 6.

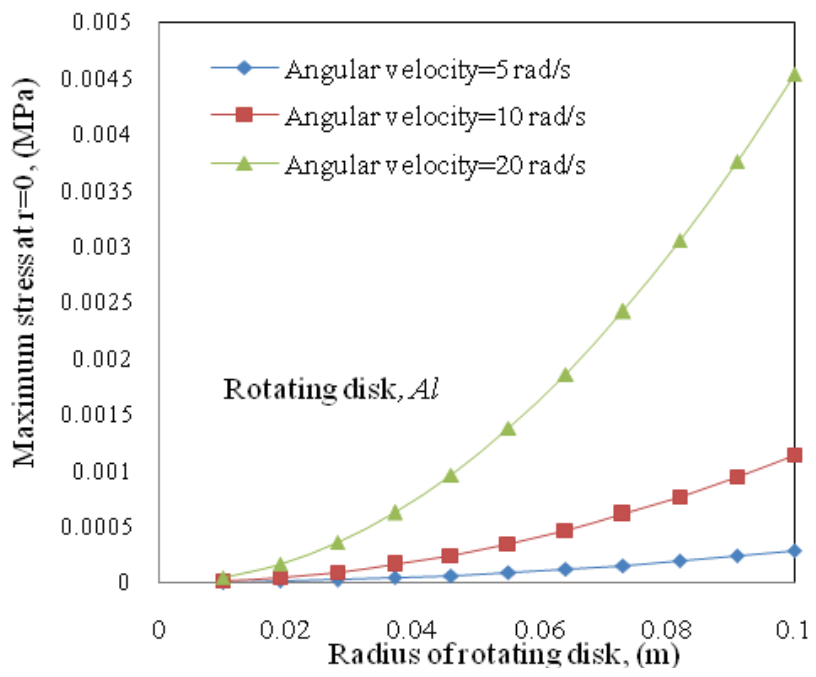

Figure 2. Maximum stress values in the rotating disk under various conditions for $\mathrm{Al}$ at $\mathrm{r}=0$.

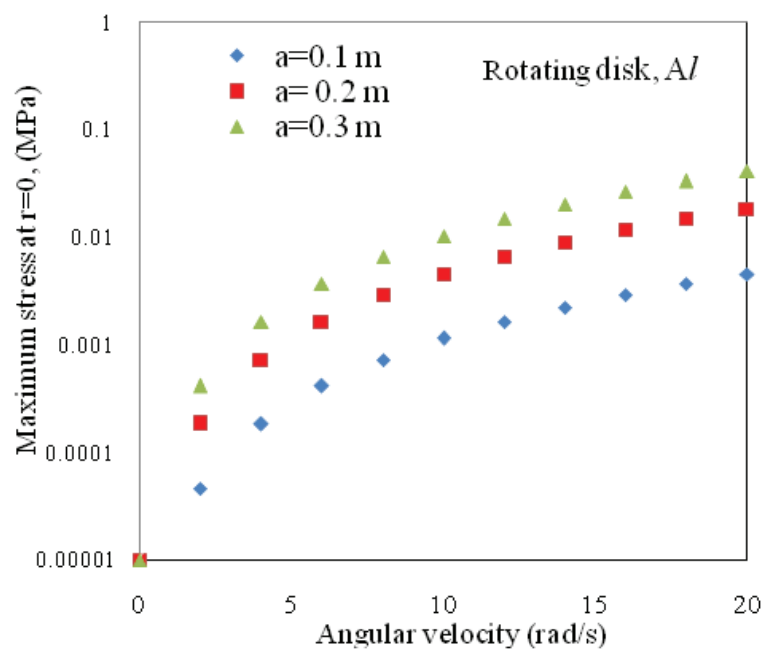

Figure 3. Maximum stress values in the rotating disk under various conditions for $\mathrm{Al}$ at $\mathrm{r}=0$.

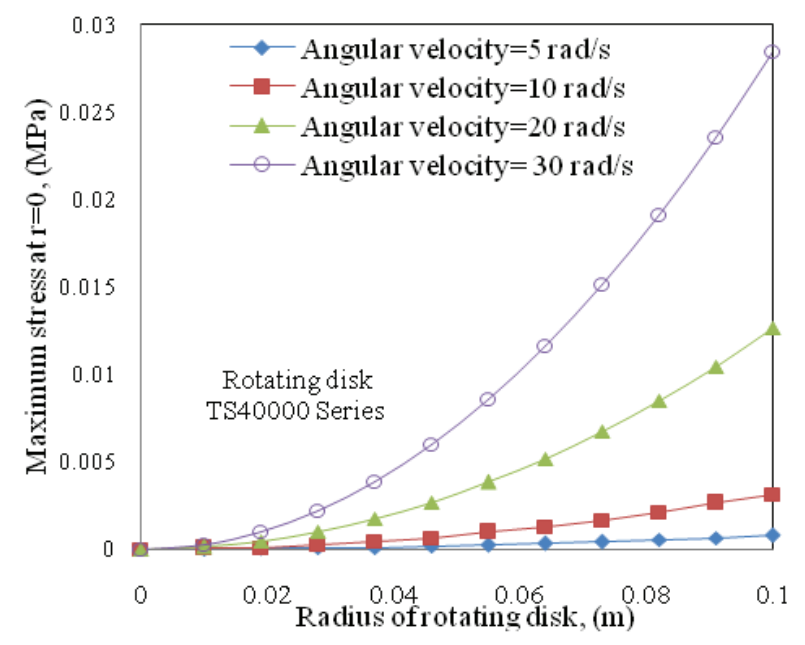

Figure 4. Maximum stresses in the rotating disk under various conditions for Steel at $r=0$.

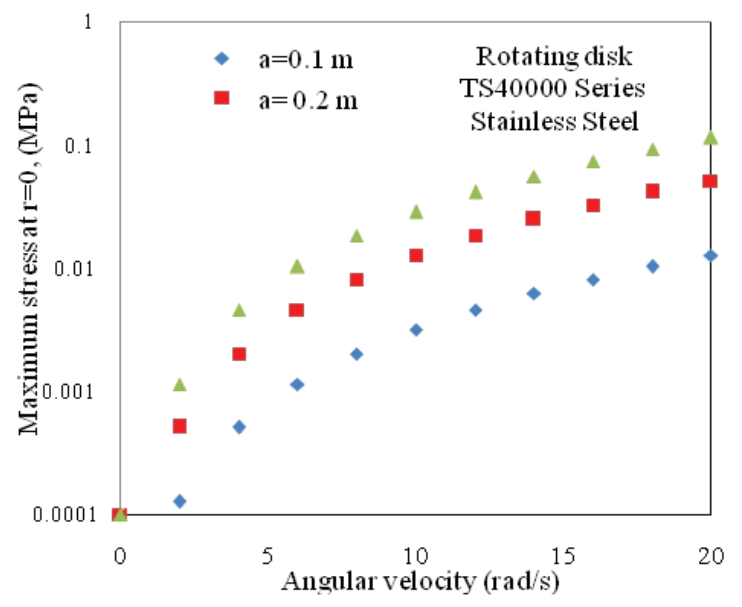

Figure 5. Maximum stress values in the rotating disk under various conditions for Steel at $r=0$. 


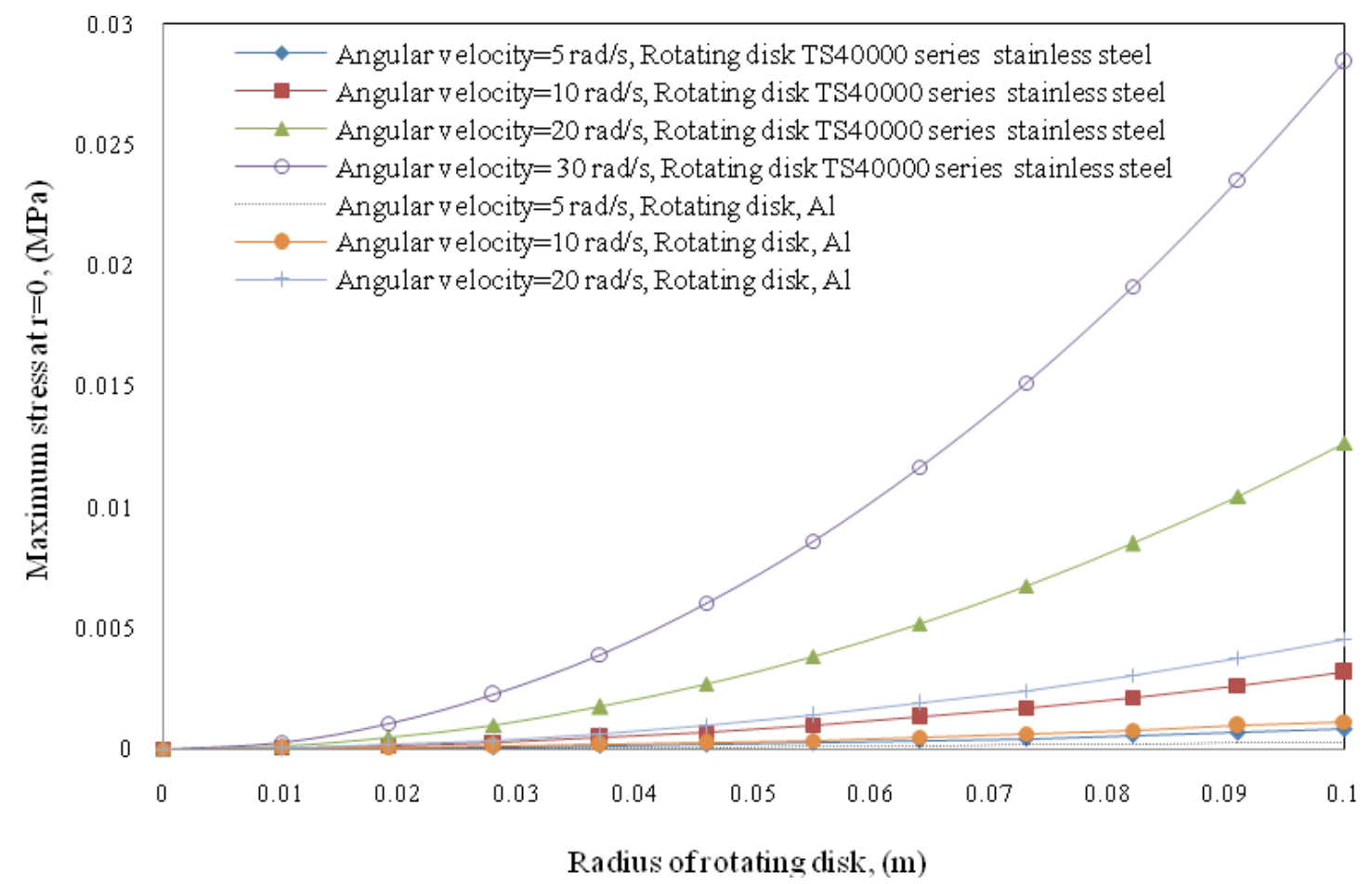

Figure 6. Comparison of maximum stress values in the rotating disk for steel and aluminum under various conditions at $\mathrm{r}=0$.

Above results show that maximum stress values in steel rotating disk with angular velocity $30 \mathrm{rad} / \mathrm{s}$ is more than the other cases. Also in similar conditions, $\omega=20 \frac{\mathrm{rad}}{\mathrm{s}}$, maximum stress values in Steel Rotating Disk is more than the other cases. Moreover, at $0<\mathrm{r}<0.02$ and for all cases, gradient of all curves are smooth and mellow in the circular rotating disk, because stress values are also approximately zero near the center of the circular rotating disk. Furthermore, stress values have very high gradients at the outer edges in the rotating disk which it can be dangerous source for defect.

\section{Conclusions}

In this research, stress behaviors of rotating disk were analyzed using equilibrium equations, geometric relations and stress functions analytically, where, Maximum Stress Values $\left(\sigma_{r, \max }, \sigma_{\theta, \max }\right)$ were determined for two different materials ( $\mathrm{A} l$, Steel) theoretically. It was observed that, stress values in the steel circular rotating disk increase with increasing radius and angular velocity. In addition, steel rotating disk has the most stress value in the center of disk. Near the center of the circular rotating disk, gradient of the stress values are approximately constant due to high withstanding at near the center of the disk. High stability of the rotating disk is arising from low gradients of the rotating disk withstanding. Sudden and abrupt changes of the stress behavior in the rotating disk have a direct relation with elastic modulus. Also, stability has a direct relation with withstanding and also has an inverse relation with stress changes and gradients and radius of the rotating disk.

Finally, all stress values and behaviors have very high gradients at the outer edges in the circular rotating disk which it is not favorable for designing machine. So, we can control stress behavior using the mentioned approach with considering parameter effects.

\section{References}

1. Ehrenfest P. Uniform rotation of rigid bodies and the theory of relativity. Physikalische Zeitschrift. 1909; vol 10(1):918.

2. Yuan ZX, Luo CJA, YAn X. Airflow pressure and shear forces on a rotating, deformed disk in an open shroud. Comm Nonlinear Sci Numer Simulat. 2004; 9(5): 481-497.

3. Bayat M, Saleem M, Sahari BB, Hamouda AMS, Mahdi E. Analysis of functionally graded rotating disks with variable thickness. Mech Res Comm. 2008; 35(5):283-309. 
4. Hojjati MH, Hassani A. Theoretical and numerical analyses of rotating discs of non-uniform thickness and density. Int J Pres Ves Pip. 2008; 85(10):694-700.

5. Hojjati $\mathrm{MH}$ and Jafari S. Semi-exact solution of elastic non-uniform thickness and density rotating disks by homotopy perturbation and Adomian's decomposition methods, Part I: elastic solution. Int J Pres Ves Pip. 2008; 85(12):871-878.

6. Turkyilmazoglu M. Analytic approximate solutions of rotating disk boundary layer flow subject to a uniform suction or injection. Int J Mech Sci. 2010; 52(12):1735-1744.

7. Turkyilmazoglu M. Purely analytic solutions of magneto hydrodynamic swirling boundary layer flow over a porous rotating disk. Computers and Fluids. 2010; 39(5):793-799.

8. Jafari S, Hojjati MH, Fathi A. Classical and modern optimization methods in minimum weight design of elastic rotating disk with variable thickness and density. Int J Pres Ves Pip. 2012; 92:41-47.

9. Peng XL and Li XF. Elastic analysis of rotating functionally graded polar orthotropic disks. Int J Mech Sci. 2012; 60(1):84-91.

10. EkhteraeiToussi H, RezaeiFarimani M. Elasto-plastic deformation analysis of rotating disc beyond its limit speed. Int J Pres Ves Pip. 2012; 89:170-177.

11. Itoh M, Yamada Y, Imao S, Gonda M. Experiments on turbulent flow due to an enclosed rotating disk. Exp Therm Fluid Sci. 1992; 5(3):359-368.

12. Gan XP, MacGregor SA. Experimental study of the flow in the cavity between rotating disks. Exp Therm Fluid Sci. 1995; 10(3):379-387.

13. Morís MA, Díez FV, Coca J. Hydrodynamics of a rotating disc contactor. Separ Purif Tech. 1997; 11(2):79-92.

14. Kolokotsa D, Yanniotis S. Experimental study of the boiling mechanism of a liquid film flowing on the surface of a rotating disc. Exp Therm Fluid Sci. 2010; 34(8):1346-1352.

15. Monfared V, Khalili MR. Investigation of relations between atomic number and composition weight ratio in PZT and SMA and prediction of mechanical behavior. Acta Physica Polonica Series A. 2011; 120(3):424-428.

16. Monfared V. A new analytical formulation for contact stress and prediction of crack propagation path in rolling bodies and comparing with Finite Element Model (FEM) results statically. Int J Phys Sci. 2011; 6(15):3613-3618.
17. Ersoy VH. An approximate solution for flow between two disks rotating about distinct axes at different speeds. Math Probl Eng. 2007; 2007:1-16.

18. Sheikholeslami M, Ashorynejad HR, Ganji DD, Kolahdooz A. Investigation of rotating MHD viscous flow and heat transfer between stretching and porous surfaces using analytical method. Math Probl Eng. 2011; 2011:1-17.

19. Sheikholeslami M, Ashorynejad HR, Domairry G, Hashim I. Flow and heat transfer of $\mathrm{Cu}$-water nano fluid between a stretching sheet and a porous surface in a rotating system. J Appl Math. 2012; 2012:1-18.

20. Pandya JU. The solution of a coupled nonlinear system arising in a three-dimensional rotating flow using spline method, Int J Math Math Sci. 2012; 2012:1-12.

21. Grote $\mathrm{KH}$ and Antonsson EK. Springer Handbook of Mechanical Engineering, Chapter 1, New York, USA: Springer Science Publications; 2009.

22. Goodarzian H, Ghobadi M, Farahabadi MA, Mohammadnezhad H, Hejazi SS. An investigation of nonlinear KdV type equations using HPM and VIM. Indian Journal of Science and Technology. 2011; 4(8):952-956.

23. Nikkhoo A, Amankhani M. Dynamic behavior of functionally graded beams traversed by a moving random load. Indian Journal of Science and Technology. 2012; 5(12):3727-3731.

24. Haghighi A R, Ghejlo $\mathrm{H} \mathrm{H}$, Asghari N. Explicit and implicit methods for fractional diffusion equations with the riesz fractional derivative. Indian Journal of Science and Technology. 2013; 6(7):4881-4885.

25. Srinivasan V. Analysis of static and dynamic load on hydrostatic bearing with variable viscosity and pressure. Indian Journal of Science and Technology. 2013; 6(6):4777-4782.

26. Anbazhagan R, Satheesh B, Gopalakrishnan K. Mathematical modeling and simulation of modern cars in the role of stability analysis. Indian Journal of Science and Technology. 2013; 6(5):4633-4641.

27. Loonker D and Banerji PK. Distributional dual series equations and fractional calculus. Indian Journal of Science and Technology. 2013; 6(1):3892-3897.

28. El-Marouf SAA. On some generalizations of the hilberthardy type integral inequalities. Indian Journal of Science and Technology. 2013; 6(2):4098-4111.

29. Available from: www.matweb.com 